\title{
Local order in AgAuCuPdPt high entropy alloy surfaces
}

Henrik H. Kristoffersen*, Jan Rossmeisl

Department of Chemistry, University of Copenhagen, 2100 København Ø, Denmark

*E-mail: hhk@chem.ku.dk

\begin{abstract}
Synthesis of high entropy alloys often involve heating precursors to above $1000^{\circ} \mathrm{C}$ such that entropy stabilization takes effect. The resulting alloys are characterized by random element distribution at the bulk lattice positions. Surfaces may however be more ordered, even at high temperatures. We explore the local order at (111), (100) and (533) surfaces of the equimolar fcc AgAuCuPdPt high entropy alloy at high temperatures and thermal equilibrium. We find that the local order is significantly increased at the (100) surface and to a lesser extend at the (533) and (111) surfaces compared to bulk AgAuCuPdPt. The (100) surface both segregates with increased amount of Au and Ag and less Pd and Pt and have a more ordered distribution of nearest neighbor atom pairs. The (111) surface segregates with increased amounts of $\mathrm{Au}$ and $\mathrm{Ag}$ and less $\mathrm{Cu}, \mathrm{Pd}$ and $\mathrm{Pt}$, but the nearest neighbor distribution is mostly random. The (100) type step edge of the (533) surface resemble the (100) surface. The degree of surface order seems linked to the structure ensemble's energy distribution width, so we suggest that the width can be used to estimate the degree of order in high entropy alloys with minimal computational efforts.
\end{abstract}


TOC

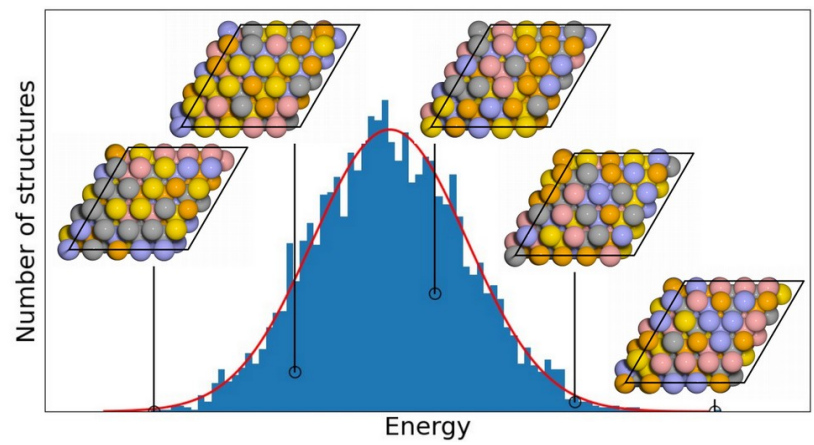




\section{Introduction}

High entropy alloys are alloys with multiple constituent elements (typically five or more) in high concentrations that can substitute freely, i.e. the constituents are randomly distributed at the bulk lattice positions. ${ }^{1-4}$ High entropy alloy catalysis has gained increasing interest, ${ }^{5-8}$ because the surfaces of high entropy alloys are expected to have all kinds of local constituent combinations and therefore likely some surface sites with high catalytic activity. ${ }^{9,10}$ However, this expectation requires that the elements in the surfaces are also randomly distributed, warranting a better understanding of how the surface composition and local surface order are related to the overall high entropy alloy composition.

High entropy alloy particles are often made by melting mixtures of metals or metal salt precursors at high temperatures $\left(\geq 1000^{\circ} \mathrm{C}\right){ }^{11-14}$ It is generally assumed that at such high temperatures the different elements are randomly distributed at the lattice sites in both bulk and at surfaces and that quenching the temperature can keep the elements in place. ${ }^{13}$ However, it is possible that some local order still exist at high temperatures, especially at surfaces, and that heating therefore will not produce randomized surfaces.

The AgAuCuPdPt high entropy alloy is catalytically active for the electrochemical $\mathrm{CO}_{2}$ and $\mathrm{CO}$ reduction reactions. ${ }^{15,16}$ We therefore choose equimolar fcc AgAuCuPdPt and study the local order in the bulk and at the (111), (100) and (533) surfaces. We construct structure ensembles with random placement of the elements for bulk and (111), (100) and (533) surface slabs. The ensembles are used to approximate the canonical partition function and obtain the local order as a function of temperature (assuming thermal equilibrium). We use slabs that have similar sized bulk and surface regions to construct the canonical ensembles, so our results will mainly apply to nanoparticles that also have similar sized bulk and surface regions.

We find that the ensemble energies are Gaussian distributed with a width (standard deviation per square root number of atoms) that increases at the surfaces as $(100)>(533)>(111)>$ bulk. The partition function effectively pics out the stable energy tail of the Gaussian distributions, so the larger 
the width of the energy distribution, the less likely it is that a randomly constructed configuration will be a good representation of the high-entropy alloy even at high temperatures. The local order therefore increases similarly to the energy distribution width with $(100)>(111)>$ bulk (the degree of local order in the (533) surface is likely in between that of (100) and (111) although we do not definitively conclude that). Two types of local order are relevant, namely the composition of elements in the surface layer (surface segregation) ${ }^{17-19}$ and the distribution of nearest neighbor elements. The (111) surface of AgAuCuPdPt segregates significantly and has increased amounts of Au and Ag in the surface layer and less $\mathrm{Cu}, \mathrm{Pd}$ and Pt. However, the nearest neighbor distribution of the elements in the (111) surface is still mostly random. The (100) surface of AgAuCuPdPt also segregates with increased amount of Au and Ag and less Pd and Pt in the surface layer. Additionally, the (100) surface has a more ordered distribution of nearest neighbor atom pairs in the surface. The (533) surface contains a (100) type step and a three atomic rows wide (111) terrace. The step edge composition is most similar to the (100) surface and the terrace is most similar to the (111) surface. We were not able to extract the nearest neighbor order in the step edge due to the limited amount of step edge sites in the (533) slab. Finally, we propose that ensemble distribution widths can be a quick way to assess whether a high entropy alloy surface will have increased order or whether it can be considered to be random.

Our most useful insight for future computational studies is that AgAuCuPdPt (111) surfaces can likely be modeled by surfaces with random atomic arrangement, where only the change in overall surface composition needs to be accounted for. This is likely not the case for AgAuCuPdPt (100) surfaces, because of their increased nearest neighbor order.

\section{Computational details}

The (111) and (100) surfaces are modeled by $p(5 \times 5)$ surface slabs with a thickness of five atomic layers (125 atoms in total). The (533) surface consists of a 4 atoms wide (111) terrace and a (100) type step. ${ }^{20}$ The (533) surface slab has five step edge atoms on each side and a thickness of five atomic 
layers (100 atoms in total). The slab and bulk computational cells are setup with the ASE program. ${ }^{21}$ The energies of the slabs are calculated with density functional theory (DFT) using the GPAW program, ${ }^{22} 400 \mathrm{eV}$ energy cutoff plane-wave basis, and spin-paired electron configuration (non spin polarized). Exchange-correlation effects are approximated by the RPBE functional. ${ }^{23}$ The (111) and (100) surface slabs are modeled with $2 \times 2 \times 1 \mathrm{k}$-points and the (533) surface slab is modeled with $2 \mathrm{k}$ points in the direction along the step and $4 \mathrm{k}$-points perpendicular to the step. The atomic regions are treated with the PAW formalism and 17, 11, 11, 16, and 16 valence electrons are included for each Ag, $\mathrm{Au}, \mathrm{Cu}, \mathrm{Pd}$, and Pt atom, respectively.

The ensemble energies $\left(\Delta E_{i}\right)$ are used to approximate the canonical partition function ( $Z$, eq 1$)$ and obtain values for local order ( $\langle A\rangle$, eq 2) as a function of temperature (assuming thermal equilibrium).

$$
\begin{aligned}
& Z \approx \sum_{i=1}^{M} \exp \left(-\Delta E_{i} /\left(k_{\mathrm{B}} T\right)\right) \\
& \langle A\rangle(T)=\frac{1}{Z} \sum_{i=1}^{M} A_{i} \exp \left(-\Delta E_{i} /\left(k_{\mathrm{B}} T\right)\right)
\end{aligned}
$$

$A_{i}$ could be the nearest neighbor distribution or the surface composition in the $i$ 'th structure of the ensemble. A weakness of our approach is that the absolute value of $Z$ is not captured, since we use a limited number of random configurations (including more configurations will always make $Z$ larger). Thermodynamic properties that require the total size of $Z$, such as the total configurational entropy ( $\left.S=k_{B} \ln Z+\langle E\rangle / T\right)$, is therefore out of reach, but properties that are given by eq 2 and dependent on how much weight certain types of configurations contribute to $Z$ can be approximated.

The energy of slab structures can be separated into bulk and surface energies. ${ }^{24}$ Only slabs with both stable bulk and stable surfaces will contribute significantly to $Z$. However, if we assume that the bulk and surface energy contributions can be treated independently, we can skip the excessive task of finding slabs with both stable bulk and surface energies and instead just find slabs with stable surface energies. We therefore choose to calculate surface energies (eq 3) and use these to construct $Z$, 
implying that we could have found slabs with the same surfaces, and the most stable bulk and gotten the same overall surface contribution to $Z$.

$\Delta E_{i}^{H E A,(111)}=E_{i}^{H E A,(111)}-E_{i}^{H E A, f c c}$

In eq $3, E_{i}^{H E A,(111)}$ is the total energy of the $i^{\prime}$ th slab and $E_{i}^{H E A, f c c}$ is the total energy of the bulk structure obtained by stacking the slab structure (we note that many different bulk structures can be obtained by sliding the stacked slabs compared to each other, but that we construct all the bulk structures in a consistent manner).

The lattice parameter for AgAuCuPdPt is approximated by the average of the DFT calculated lattice parameters for the pure metals. I.e. we apply Vegard's law even though it mostly has been validated for binary compounds., ${ }^{9,25,26}$ We relax all the atomic structures, but neglect zero point and vibrational energies, which we do not expect will deviate significantly between structures within the ensembles.

Finally, since we only construct partial partition functions the low temperature limit cannot be trusted. We designate two temperature regions, namely a low temperature "poor statistics” region where $\exp \left(-\Delta E_{\min } /\left(k_{\mathrm{B}} T\right)\right) / Z>0.25$ and a high temperature "better statistics" region where $\exp \left(-\Delta E_{\min } /\left(k_{\mathrm{B}} T\right)\right) / Z<0.25$, separating where the most stable ensemble structure weigh more or less than $25 \%$ in the partition function. We disregard the possibility that we may exceed the melting point of the AgAuCuPdPt alloy. The melting points of the constituents are Ag; $1234 \mathrm{~K}$, Au; $1336 \mathrm{~K}, \mathrm{Cu} ; 1356$ K, Pd; $1825 \mathrm{~K}$, Pt $2042 \mathrm{~K} .{ }^{27}$ Our rational is that if the solid possesses order at very high temperatures, where it is unstable compared to the molten alloy, it will be even more ordered at lower temperatures.

The ensemble structures, DFT calculations, and python scripts that are the basis for this study can be accessed online at https://nano.ku.dk/english/research/theoretical-electrocatalysis/katladb/localorder/ . 


\section{Results and discussion}

\subsection{Local order in bulk AgAuCuPdPt}

We calculated 10100 bulk structures of the form $\mathrm{Ag}_{25} \mathrm{Au}_{25} \mathrm{Cu}_{25} \mathrm{Pd}_{25} \mathrm{Pt}_{25}$, where the 125 atoms were randomly shuffled (6000 are the reference bulk structures used for the (100) study and 4000 are the reference bulk structures used for the (111) study). The energies of the bulk structures are referenced to pure bulk metals and their distribution is plotted in Figure 1a. The energies are Gaussian distributed with an average of $-4.41 \mathrm{eV}$, however, several structures have energies below $-5.5 \mathrm{eV}$ (insert Figure 1a) and the most stable structure is $-5.93 \mathrm{eV}$. The distribution has a width per square root number of atoms of $0.0317 \mathrm{eV}$ (Table 1), which is much smaller than the surfaces that we will discuss in the subsequent sections. Due to the nature of the partition function, the most stable structure dominates at low temperatures and the temperate has to be above $1496 \mathrm{~K}$ before it weighs less than $25 \%$. The ensemble energies are well below $0 \mathrm{eV}$, so the equimolar fcc AgAuCuPdPt high entropy alloy is more stable than the pure metals of its constituents even without entropy being taken into account. We note that AgAuCuPdPt could still be unstable, since we have not compared the energy of AgAuCuPdPt to all the viable alloy combinations. ${ }^{1}$

It is possible for bulk AgAuCuPdPt to have internal order, even though the structure ensemble is constructed from random structures, if the most stable structures happen to have internal order. To quantify the degree of internal order we calculate the nearest neighbor order parameter $\alpha_{i j}$ (eq 4). ${ }^{28,29}$

$\alpha_{i j}=1-\frac{\left\langle n_{i j}\right\rangle}{x_{j} \cdot N}$

In eq $4,\left\langle n_{i j}\right\rangle$ is the average number of $j$ atoms at the nearest neighbor sites next to an $i$ atom, $N$ is the number of nearest neighbor sites (equal to 12 in the bulk) and $x_{j}$ is the overall molar fraction of $j$ atoms. ${ }^{28}$ Since we know that the $i$ atom cannot be neighbor to itself, we adjust $x_{j}$ to match the remaining molar fraction (i.e. $x_{j}$ is $25 / 124$ for $i \neq j$ and $24 / 124$ for $i=j$ ). A random distribution of nearest neighbor atom pairs $(i, j)$ gives $\alpha_{i j}=0$, low affinity for $i, j$ pairs gives $\alpha_{i j}>0$ and high affinity for $i, j$ pairs gives 
$\alpha_{i j}<0$. Selected nearest neighbor order parameters are plotted in Figure 1b and show that bulk AgAuCuPdPt has reduced affinity for e.g. Pt-Au and Pd-Pd pairs and extra affinity for e.g. Cu-Pt and Pd-Au pairs (The full set of nearest neighbor order parameters are included in the Supporting Information). The degree of nearest neighbor order in AgAuCuPdPt at high temperatures is similar to other multi component alloys, such as bulk bcc NbMoTaW, ${ }^{30}$ bulk fcc VCoNi, ${ }^{31}$ and bulk fcc CrFeCoNi. ${ }^{32}$
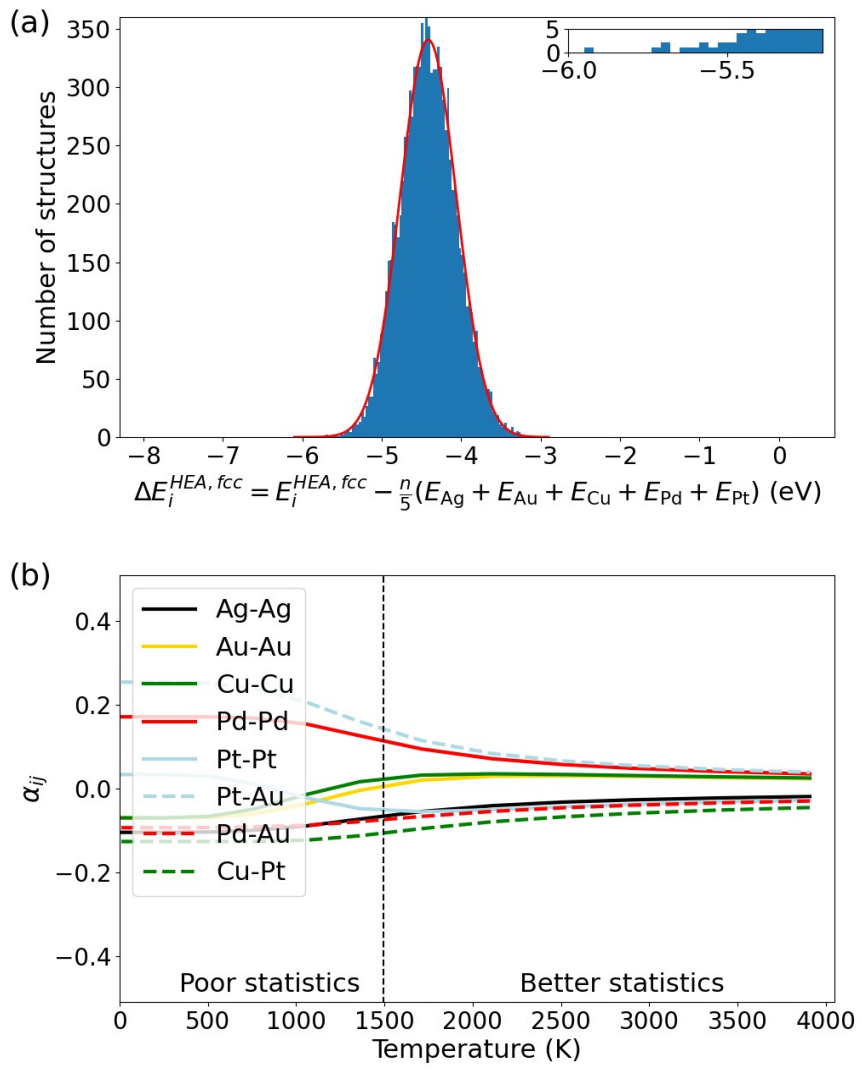

Figure 1: (a) Energy distribution of 10100 bulk AgAuCuPdPt structures with 125 atoms. A Gaussian is constructed from the number of structures, average energy, and standard deviation of the distribution and plotted in red. (b) Selected order parameters $\left(\alpha_{i j}\right)$ for nearest neighbor atom pairs in bulk AgAuCuPdPt. The most stable structure weighs more than 25\% in the "poor statistics” region. 
Table 1: Width of the energy distributions (standard deviation per square root number of atoms $(n)$ ). Both the width of the total energy distribution $(\sigma(E))$ and the width of the surface energy distribution $(\sigma(\Delta E))$ are tabulated.

System

Bulk
$\sigma(E) / \sqrt{n}$
$\sigma(\Delta E) / \sqrt{n}$
$0.0317 \mathrm{eV}$
$0.0861 \mathrm{eV}$
$0.0813 \mathrm{eV}$
$0.1138 \mathrm{eV}$
$0.1102 \mathrm{eV}$
$0.0906 \mathrm{eV}$
$0.0853 \mathrm{eV}$

\subsection{Local order in AgAuCuPdPt (111) surfaces}

We calculated 4000 AgAuCuPdPt (111) slabs containing 125 randomly shuffled atoms and subtracted the energy of bulk systems with the same atomic placement to obtain surface energies. The width of the surface energy distribution (Figure 2a) is much larger than the bulk energy distribution (0.0813 eV per squire root number of atoms, Table 1). This will inevitably result in fewer structures contributing substantially to the partition function and likely a higher degree of order. The distribution width of the total slab energies without subtracting bulk energies is $0.0861 \mathrm{eV}$ per squire root number of atoms, and therefore only a little wider than the surface energy distribution. The slab with the most stable (111) surfaces configuration are enriched in Au and Ag at the expense of Pd, Pt and $\mathrm{Cu}$ (Figure 2b). The top surface has 9Ag, 6Au, 3Cu, Pd, and 6Pt, the bottom surface has 2Ag, 10Au, 4Cu, 6Pd, and 3Pt. 

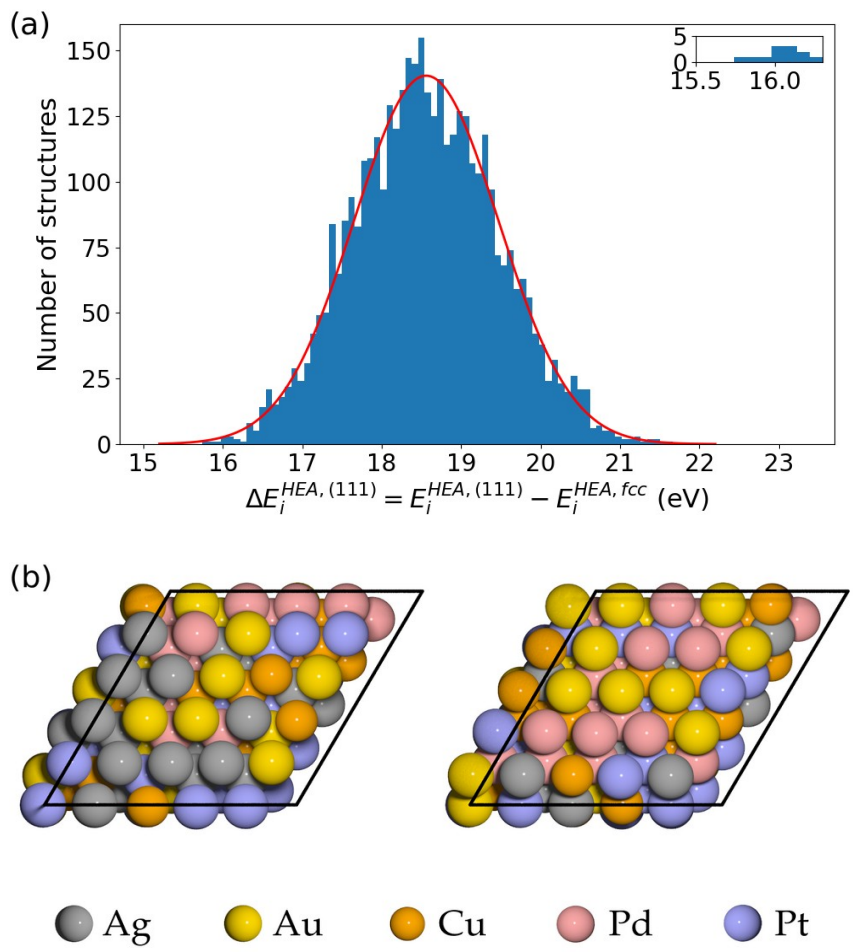

Figure 2: (a) Surface energy distribution of 4000 AgAuCuPdPt (111) slabs with 125 atoms. A Gaussian is constructed from the number of structures, average energy, and standard deviation of the distribution and plotted in red. (b) Top and bottom surfaces of the most stable (111) surface configuration.

The AgAuCuPdPt (111) surface composition is shown in Figure 3a as a function of temperature. The figure shows that the modification of the surface composition found in the most stable slab is maintained even at very high temperature. For instance, at $2000 \mathrm{~K}$ the (111) surface is expected to contain 30\% Au, 23\% Ag, 17\% Pd, 16\% Pt and 13\% Cu rather than the $20 \%$ of each element found in the overall composition. The surface is therefore significantly segregated. The surface composition generally follows what would be expected from the pure metal (111) surface energies with Au(111) having the most stable surface energy (0.25 eV per surface atom) and $\mathrm{Pt}(111)$ having the least stable (0.56 eV per surface atom). Pure metal surface energies are shown in Table 2 and are similar to previous calculations. ${ }^{24,33}$ The most stable AgAuCuPdPt (111) surface configuration has a surface energy of $0.32 \mathrm{eV}$ per atom, which is a little lower than what would be expected from weighing the 
pure metal surface energies with the surface composition found in the most stable (111) surface configuration (giving $0.36 \mathrm{eV} / \mathrm{atom})$.

Table 2: Surface energies of pure metals and the most stable (0 K limit) AgAuCuPdPt (111), (100), and (533) surface configurations (eV/ $\left.n_{\text {surf }}\right)$.

(111)

Ag

Au

$\mathrm{Cu}$

Pd

Pt

AgAuCuPdPt (0 K)
0.27

0.25

0.40

0.48

0.56

0.32
(100)

0.34

0.36

0.51

0.64

0.80

0.44 $(533)^{a}$

0.29

0.26

0.42

0.51

0.58

0.33

${ }^{a} n_{\text {surf }}{ }^{(533)}$ is 40 with 10 step edge atoms and 30 terrace atoms.

Cu stands out by being the least abundant element in the (111) surface, but having a middle of the range surface energy. This can be explained by $\mathrm{Cu}$ being favored in the second layer, where many of the $\mathrm{Cu}$ atoms missing in the surface layer are found instead (25\% $\mathrm{Cu}$ at $2000 \mathrm{~K}$, Figure 2b). A similar preference for $\mathrm{Cu}$ in the subsurface region instead of in the surface layer has already been observed for Pt-Cu alloys. ${ }^{34,35} \mathrm{Pt}$ is preferred in the inner most layer ( ${ }^{\text {rd }}$ layer) with $26 \%$ Pt at $2000 \mathrm{~K}$ (Figure 2c). On the hand, $\mathrm{Au}$ is severely depleted from the $2^{\text {nd }}$ layer $(13 \% \mathrm{Au}$ at $2000 \mathrm{~K})$ and $3^{\text {rd }}$ layer $(13 \% \mathrm{Au}$ at $2000 \mathrm{~K}$ ) due to the preference for the surface. It has similarly been found computationally ${ }^{36}$ that a equimolar fcc CoNiCrFeMn (the so-called Cantor alloy) slab with 20 atomic layers has a depletion of $\mathrm{Mn}$ in the bulk due to a build up in the (100) surface layer and a depletion of Cr in the bulk due to a preference for the near surface region. Mn also has the lowest (100) surface energy of all the 
CoNiCrFeMn constituents. Another computational study investigated the CoNiCrFeMn (111) surface and found that Ni will segregate to the surface. ${ }^{37}$

To further study the local order in the AgAuCuPdPt (111) surface we calculate the nearest neighbor order parameter $\left(\alpha_{i j}^{\text {surf }}\right)$ for the atoms in the (111) surface.

$\alpha_{i j}^{\text {surf }}=1-\frac{\left\langle n_{i j}^{\text {surf }}\right\rangle}{x_{j}^{\text {surf }} \cdot N^{\text {surf }}}$

$\left\langle n_{i j}^{\text {surf }}\right\rangle$ is the average number of $j$ atoms at the nearest neighbor surface sites next to an $i$ atom, $N^{\text {surf }}$ is the number of nearest neighbor surface sites (6 for (111)) and $x_{j}^{\text {surf }}$ is the molar fraction of $j$ atoms in the surface. At high temperatures the distribution of nearest neighbor elements in the (111) surface is only a little more ordered than in the bulk (Figure 3d). Most nearest neighbor pairs that are favored in the (111) surface (such as Pt-Pt, Pd-Au and Cu-Pt) are likewise favored in the bulk (Figure 1b), and most atomic pairs that are disfavored in the (111) surface (such as $\mathrm{Cu}-\mathrm{Cu}$ and $\mathrm{Pt}-\mathrm{Au}$ ) are also disfavored in the bulk. The main exception is that the number of Pd-Pd pairs in the surface is higher than what would be expected from the Pd surface concentration and a random distribution. This is unexpected since nearest neighbor Pd-Pd pairs are unfavored in the bulk, but maybe it can be explained by Pd's affinity for $\mathrm{Au}$, which causes Pd to also sit next to other Pd atoms in order to optimize their overall presence next to Au atoms. The full set of nearest neighbor order parameters for the (111) surface is included in the Supporting Information.
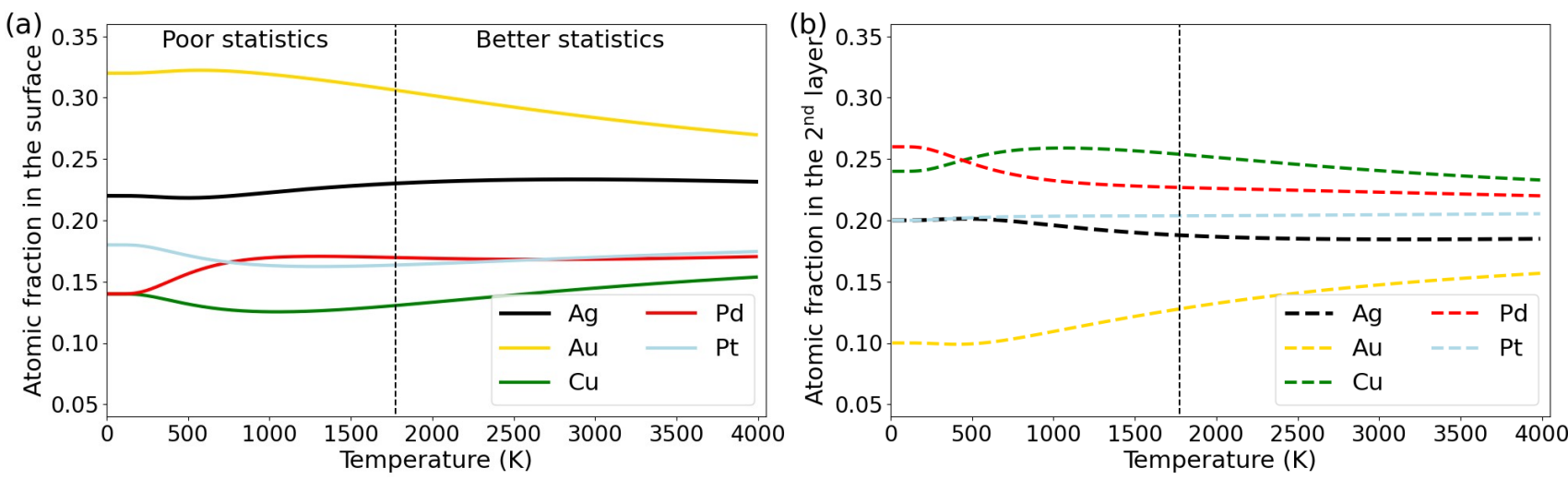

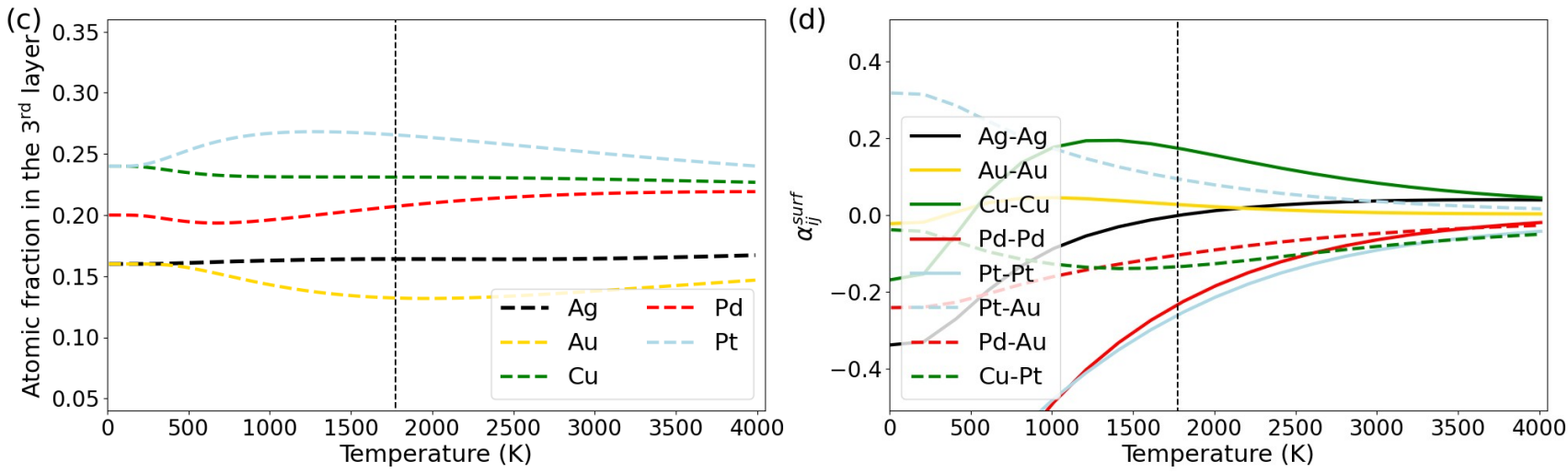

Figure 3: Element composition in the (a) (111) surface layer, (b) $2^{\text {nd }}$ layer, and (c) $3^{\text {rd }}$ layer as a function of temperature. (d) Selected order parameters $\left(\alpha_{i j}^{\text {sur }}\right)$ for nearest neighbor pairs in the AgAuCuPdPt (111) surface.

\subsection{Local order in AgAuCuPdPt (100) surfaces}

We calculated 6000 AgAuCuPdPt (100) slabs containing 125 randomly shuffled atoms and subtracted the energies of the corresponding bulk. The width $(0.1102 \mathrm{eV}$, Table 1$)$ of the surface energy distribution (Figure 4a) is both larger than the bulk and the (111) energy distribution. The width is actually so large that we get very little contribution to the partition function from other structures than the most stable ones, and we therefore chose to calculate 6000 structures rather than 4000 to improve the statistics. Still, the most stable (100) configuration contributes more than $25 \%$ to $Z$ at temperatures below $3577 \mathrm{~K}$. The top surface of the most stable (100) configuration contains 8Ag, 7Au, 6Cu, 2Pd, 2Pt, whereas the bottom surface contain 5Ag, 9Au, 5Cu, 3Pd, 3Pt (Figure 4b). 


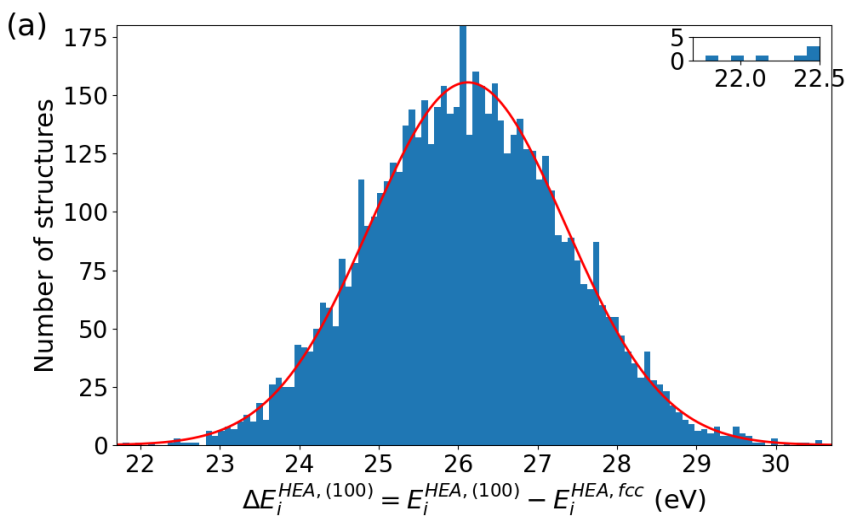

(b)
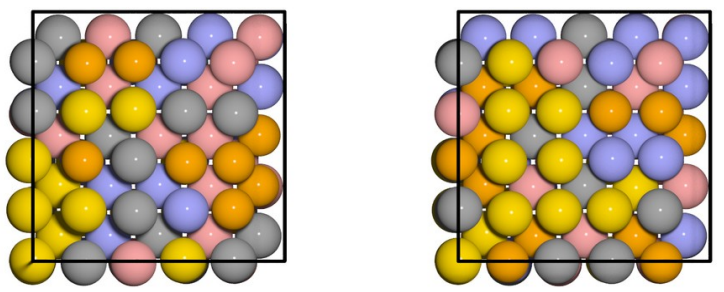

- Ag $\odot \mathrm{Au} \odot \mathrm{Cu} \odot \mathrm{Pd} \odot \mathrm{Pt}$

Figure 4: (a) Surface energy distribution of 6000 AgAuCuPdPt (100) slabs with 125 atoms. A Gaussian is constructed from the number of structures, average energy, and standard deviation of the distribution and plotted in red. (b) Top and bottom surfaces of the most stable (100) surface configuration.

The temperature dependent AgAuCuPdPt (100) surface composition is shown in Figure 5a and has many similarities to the composition of the (111) surface with Au (and to a lesser extend Ag) being preferred and Pd and Pt being disfavored. The main difference is that $\mathrm{Cu}$ is not nearly as scarce in the (100) surface as it is in the (111) surface, which in turn means that the Cu concentration is also not particular enhanced in the $2^{\text {nd }}$ (Figure 5b) or $3^{\text {rd }}$ (Figure 5c) layer. The concentration of the elements in the surface still doesn't completely follow the pure element surface energies (Table 2), since Ag(100) has a more stable surface energy than $\mathrm{Au}(100)$, while $\mathrm{Au}$ is more abundant than Ag in the AgAuCuPdPt (100) surface. The most stable AgAuCuPdPt (100) surface configuration has a surface energy of 0.44 
$\mathrm{eV}$ per atom (compared to $0.46 \mathrm{eV}$ per atom from weighing the pure metal surface energies with the surface composition).

We again use eq 5 to calculate the nearest neighbor order parameters for the atoms in the (100) surface $\left(N^{\text {surf }}=4\right.$ for $\left.(100)\right)$ and show a selection of them in Figure $5 \mathrm{~d}$. The figure shows that the order parameters deviate significantly from 0 , and that the surface has a more ordered distribution of nearest neighbor atom pairs compared to both the (111) surface and bulk AgAuCuPdPt. In the (100) surface, Pt-Pt pairs and Pt-Cu pairs are present in much larger abundance than expected if the atoms in the surface were randomly placed. Oppositely, Pd-Pd nearest neighbor pairs are very uncommon, even though the order parameter accounts for the low Pd surface concentration. The increased presence or increased lack of certain nearest neighbor pairs could significantly influence the catalytic properties of the AgAuCuPdPt (100) surface., ${ }^{9,38,39}$ The full set of nearest neighbor order parameters for the (100) surface is included in the Supporting Information.
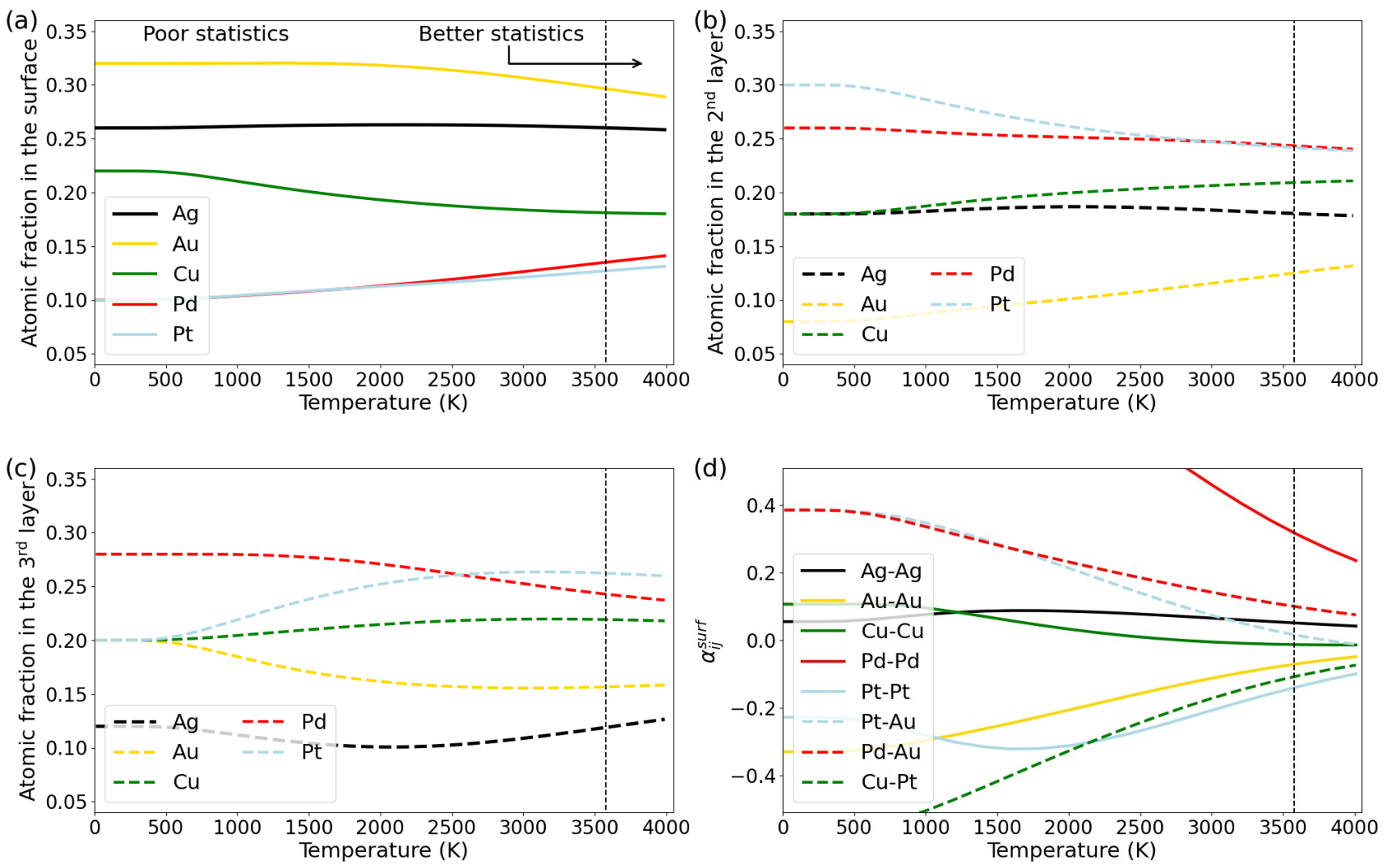
Figure 5: Element composition in the (a) (100) surface layer, (b) $2^{\text {nd }}$ layer, and (c) $3^{\text {rd }}$ layer as a function of temperature. (d) Selected order parameters $\left(\alpha_{i j}^{\text {surf }}\right)$ for nearest neighbor pairs in the AgAuCuPdPt (100) surface.

\subsection{Local order in AgAuCuPdPt (533) steps}

Finally, we calculated 4000 AgAuCuPdPt (533) slabs containing 100 randomly shuffled atoms and subtracted the energies of the corresponding bulk. The overall width of the surface energy distribution (Figure 6a) is dependent on the number of atoms in the simulation, so we divide the width by the squire root number of atoms such that it can be compared to the ensembles with 125 atoms. The distribution has a width per square root number of atoms of $0.0853 \mathrm{eV}$ (Table 1), which is in between the width of the (111) ensemble and the (100) ensemble. The most stable (533) configuration has $2 \mathrm{Ag}, 2 \mathrm{Cu}$, and 1 $\mathrm{Pd}$ atom in the step edge of the top surface and $3 \mathrm{Au}, 1 \mathrm{Ag}$, and $1 \mathrm{Cu}$ at the step edge of the bottom surface (Figure 6b).

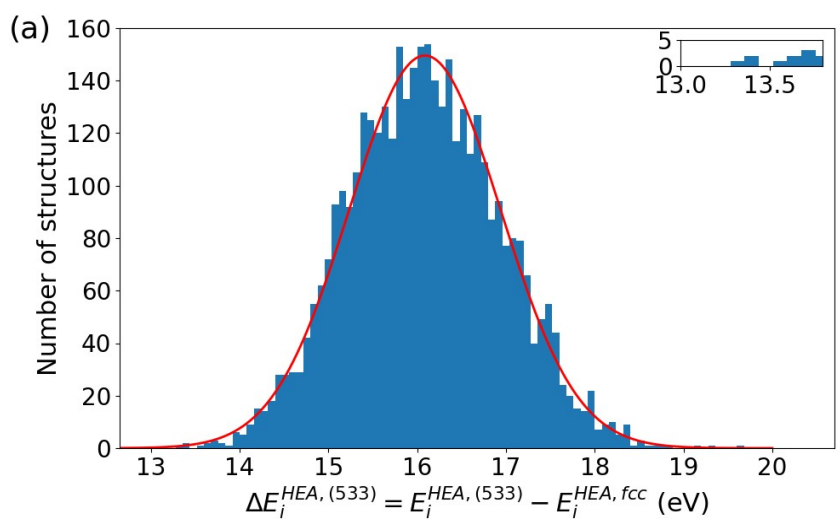

(b)
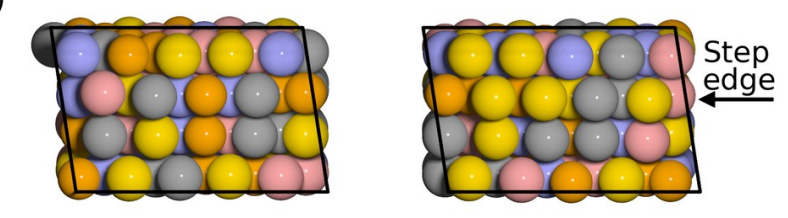

- Ag $\mathrm{Au} \bigcirc \mathrm{Cu} \bigcirc \mathrm{Pd} \bigcirc \mathrm{Pt}$ 
Figure 6: (a) Surface energy distribution of 4000 AgAuCuPdPt (533) slabs with 100 atoms. A Gaussian is constructed from the number of structures, average energy, and width of the distribution and plotted in red. (b) Top and bottom surfaces of the most stable (533) surface configuration.

The element composition at the step edge as a function of temperature is shown in Figure 7a. The step edge composition resembles most closely the (100) surface composition, with Au (32\% at $2000 \mathrm{~K}$ ) and $\mathrm{Ag}(32 \%$ at $2000 \mathrm{~K})$ being most abundant and $\mathrm{Cu}(18 \%$ at $2000 \mathrm{~K})$ being significantly more abundant than Pd (11\% at $2000 \mathrm{~K})$ and Pt (7\% at $2000 \mathrm{~K})$. The element composition in the terrace (the three rows of atoms between step atoms) are shown in Figure 7b and most closely resemble the (111) surface with $\mathrm{Au}(31 \%$ at $2000 \mathrm{~K})$ being most abundant and Pt (16\% at $2000 \mathrm{~K})$, $\mathrm{Cu}(16 \%$ at $2000 \mathrm{~K})$ and Pd (14\% at $2000 \mathrm{~K})$ being most scarce. The step edge at the (533) surface is of the (100) type meaning that the step edge atoms and the adjacent lower terrace atoms sit in a squire pattern similar to the (100) surface, explaining why the composition of the step edge atoms differ from composition of the terrace atoms which sit in a (111) surface configurations.

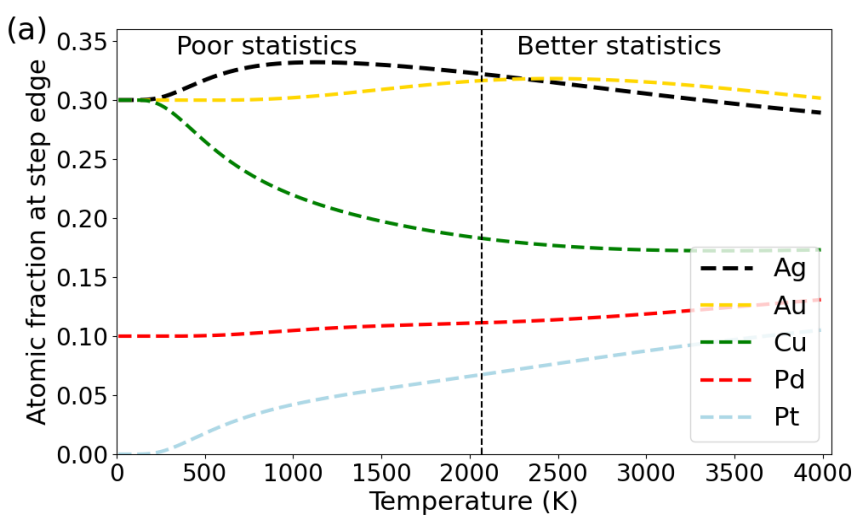




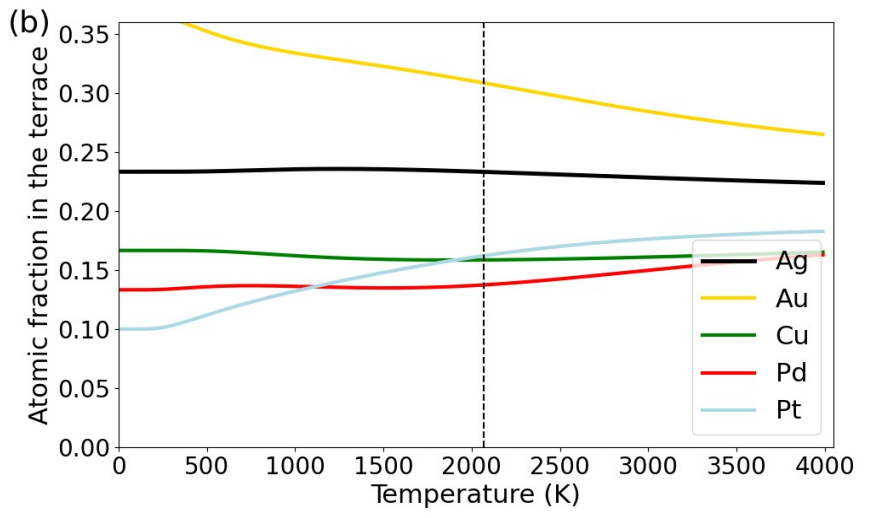

Figure 7: Element composition at the (a) (533) step edge and (b) in the (533) terrace as a function of temperature.

\subsection{Estimating local order in high entropy alloys}

High entropy alloys are generally considered to be random. However, it is actually unlikely that a random structure (with 125 atoms) will be a good representation of the AgAuCuPdPt high entropy alloy. For instance, at $1500 \mathrm{~K}$ the 505 most stable structures (5\%) in the bulk AgAuCuPdPt ensemble make up $88 \%$ of $Z$, indicating that the remaining 9595 structures (95\%) are not particularly representative of the bulk AgAuCuPdPt high entropy alloy. This is further exaggerated in the surface slab ensembles due to the increased width of the energy distributions. For instance, at $1500 \mathrm{~K}$ the 40 most stable structures (1\%) in of the AgAuCuPdPt (111) surface ensemble make up 97\% of $Z$.

It requires several thousand ensemble structures to get a decent representation of $Z$, and from that an understanding of the local order in the bulk and at the surfaces of high entropy alloys. However, if the ensemble energy width is linked to the degree of order, the width could be a useful indicator to check whether local order should be accounted for in the first place. Figure 8 shows the standard deviation per square root number of atoms of the ensemble energies as a function of number of structures in the ensembles. At around 40 structures the standard deviation is largely converged, so 
obtaining an estimate of the degree of local order from the ensemble width is relative computational inexpensive.

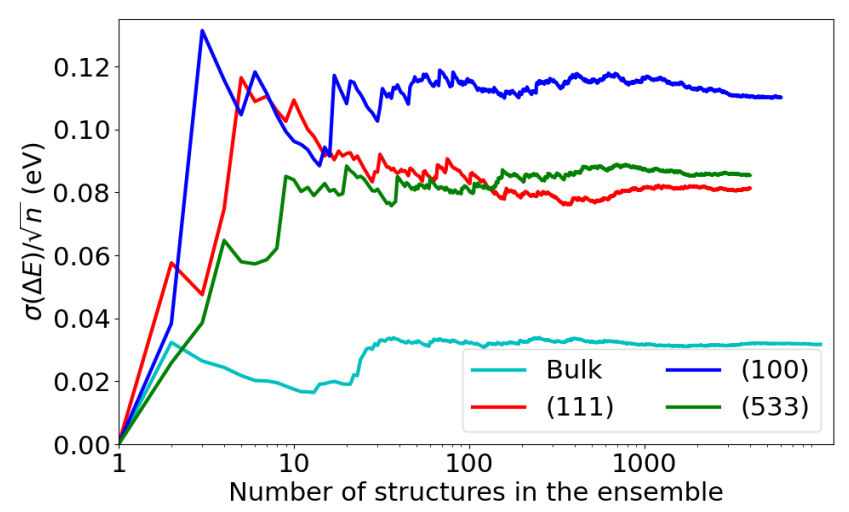

Figure 8: Standard deviation per square root number of atoms of the ensemble energy distributions plotted as a function of the number of structures in the ensembles.

\section{Conclusion}

Our study shows that the surface atoms in the equimolar fcc AgAuCuPdPt high entropy alloy influence the total energy significantly more than atoms in the bulk. The energy distribution of the randomized structure ensembles for the (100), (533), and (111) surface slabs are therefore much wider than the randomized structure ensemble for the bulk. Consequently, only the surface structures with energies in the stable tail of the energy ensembles will be present even at high temperature and they give rise to local order in the AgAuCuPdPt surfaces.

One type of local order is surface segregration. All the studied surfaces segregate to have increased concentration of Au and Ag. This can largely be explained by the low surface energy of these metals, however $\mathrm{Cu}$ is more scarce in the AgAuCuPdPt (111) surface than would be expected from its surface energy, because there is a preference for $\mathrm{Cu}$ in the second layer. Furthermore, nearest neighbor atomic 
order seems to play a role in the stability of the AgAuCuPdPt (100) surface, and the precise placement of the (100) surface atoms is therefore unlikely to be random.

Surface structures with energies that lie further down the tail of the energy ensembles will contribute exponentially more to the canonical partition function. It therefore seems resolvable to link the width of the structure ensemble energy distribution to the degree of local order. This is convenient because obtaining an estimate of the structure ensemble energy distribution width is computationally inexpensive and could therefore be used to assess whether local order should be accounted for in any given high entropy alloy.

\section{Acknowledgements}

The authors acknowledge support from the Danish National Research Foundation Center for High-Entropy Alloy Catalysis (CHEAC) DNRF-149.

\section{Supporting Information}

Full set of nearest neighbor order parameters for bulk AgAuCuPdPt and for the surface atoms in the AgAuCuPdPt (111) and AgAuCuPdPt (100) surfaces.

\section{References}

(1) Widom, M. Modeling the Structure and Thermodynamics of High-Entropy Alloys. J. Mater. Res. 2018, 33 (19), 2881-2898.

(2) George, E. P.; Raabe, D.; Ritchie, R. O. High-Entropy Alloys. Nat. Rev. Mater. 2019, 4 (8), 515534.

(3) Pickering, E. J.; Jones, N. G. High-Entropy Alloys: A Critical Assessment of Their Founding Principles and Future Prospects. Int. Mater. Rev. 2016, 61 (3), 183-202. 
(4) Tsai, M.-H.; Yeh, J.-W. High-Entropy Alloys: A Critical Review. Mater. Res. Lett. 2014, 2 (3), 107-123.

(5) Ferrari, A.; Dutta, B.; Gubaev, K.; Ikeda, Y.; Srinivasan, P.; Grabowski, B.; Körmann, F. Frontiers in Atomistic Simulations of High Entropy Alloys. J. Appl. Phys. 2020, 128 (15), 150901.

(6) Feng, J.; Chen, D.; Pikhitsa, P. V; Jung, Y.; Yang, J.; Choi, M. Unconventional Alloys Confined in Nanoparticles: Building Blocks for New Matter. Matter 2020, 3 (5), 1646-1663.

(7) Löffler, T.; Savan, A.; Garzón-Manjón, A.; Meischein, M.; Scheu, C.; Ludwig, A.; Schuhmann, W. Toward a Paradigm Shift in Electrocatalysis Using Complex Solid Solution Nanoparticles. ACS Energy Lett. 2019, 4 (5), 1206-1214.

(8) Ma, Y.; Ma, Y.; Wang, Q.; Schweidler, S.; Botros, M.; Fu, T.; Hahn, H.; Brezesinski, T.; Breitung, B. High-Entropy Energy Materials: Challenges and New Opportunities. Energy Environ. Sci. 2021, 14 (5), 2883-2905.

(9) Batchelor, T. A. A.; Pedersen, J. K.; Winther, S. H.; Castelli, I. E.; Jacobsen, K. W.; Rossmeisl, J. High-Entropy Alloys as a Discovery Platform for Electrocatalysis. Joule 2019, 3 (3), 834-845.

(10) Pedersen, J. K.; Batchelor, T. A. A.; Yan, D.; Skjegstad, L. E. J.; Rossmeisl, J. Surface Electrocatalysis on High-Entropy Alloys. Curr. Opin. Electrochem. 2021, 26, 100651.

(11) Yeh, J.-W.; Chen, S.-K.; Lin, S.-J.; Gan, J.-Y.; Chin, T.-S.; Shun, T.-T.; Tsau, C.-H.; Chang, S.-Y. Nanostructured High-Entropy Alloys with Multiple Principal Elements: Novel Alloy Design Concepts and Outcomes. Adv. Eng. Mater. 2004, 6 (5), 299-303.

(12) Kumar, N.; Tiwary, C. S.; Biswas, K. Preparation of Nanocrystalline High-Entropy Alloys via Cryomilling of Cast Ingots. J. Mater. Sci. 2018, 53 (19), 13411-13423.

(13) Yonggang, Y.; Zhennan, H.; Pengfei, X.; D., L. S.; Jiji, J. R.; Hua, X.; Fengjuan, C.; Anmin, N.; Tiancheng, P.; Miles, R.; et al. Carbothermal Shock Synthesis of High-Entropy-Alloy Nanoparticles. Science 2018, 359 (6383), 1489-1494.

(14) Riva, S.; Brown, S. G. R.; Lavery, N. P.; Tudball, A.; Yusenko, K. V. Spark Plasma Sintering of High Entropy Alloys BT - Spark Plasma Sintering of Materials: Advances in Processing and Applications; Cavaliere, P., Ed.; Springer International Publishing: Cham, 2019; pp 517-538.

(15) Pedersen, J. K.; Batchelor, T. A. A.; Bagger, A.; Rossmeisl, J. High-Entropy Alloys as Catalysts for the CO2 and CO Reduction Reactions. ACS Catal. 2020, 10 (3), 2169-2176.

(16) Nellaiappan, S.; Katiyar, N. K.; Kumar, R.; Parui, A.; Malviya, K. D.; Pradeep, K. G.; Singh, A. K.; Sharma, S.; Tiwary, C. S.; Biswas, K. High-Entropy Alloys as Catalysts for the CO2 and CO Reduction Reactions: Experimental Realization. ACS Catal. 2020, 10 (6), 3658-3663.

(17) Abraham, F. F.; Brundle, C. R. Surface Segregation in Binary Solid Solutions: A Theoretical and Experimental Perspective. J. Vac. Sci. Technol. 1981, 18 (2), 506-519. 
(18) Burton, J. J.; Machlin, E. S. Prediction of Segregation to Alloy Surfaces from Bulk Phase Diagrams. Phys. Rev. Lett. 1976, 37 (21), 1433-1436.

(19) Ferrari, A.; Körmann, F. Design of Compositionally Complex Catalysts: Role of Surface Segregation. J. Mater. Res. Technol. 2021, 14, 1830-1836.

(20) Backus, E. H. G.; Eichler, A.; Grecea, M. L.; Kleyn, A. W.; Bonn, M. Adsorption and Dissociation of NO on Stepped Pt (533). J. Chem. Phys. 2004, 121 (16), 7946-7954.

(21) Larsen, A.; Mortensen, J.; Blomqvist, J.; Castelli, I.; Christensen, R.; Dulak, M.; Friis, J.; Groves, M.; Hammer, B.; Hargus, C.; et al. The Atomic Simulation Environment-a Python Library for Working with Atoms. J. Phys. Condens. Matter 2017, 29 (27), 273002.

(22) Enkovaara, J.; Rostgaard, C.; Mortensen, J. J.; Chen, J.; Dułak, M.; Ferrighi, L.; Gavnholt, J.; Glinsvad, C.; Haikola, V.; Hansen, H. A.; et al. Electronic Structure Calculations with GPAW: A Real-Space Implementation of the Projector Augmented-Wave Method. J. Phys. Condens. Matter 2010, 22 (25).

(23) Hammer, B.; Hansen, L. B.; Nørskov, J. K. Improved Adsorption Energetics within DensityFunctional Theory Using Revised Perdew-Burke-Ernzerhof Functionals. Phys. Rev. B 1999, 59 (11), 7413-7421.

(24) Singh-Miller, N. E.; Marzari, N. Surface Energies, Work Functions, and Surface Relaxations of Low-Index Metallic Surfaces from First Principles. Phys. Rev. B 2009, 80 (23), 235407.

(25) Vegard, L. Die Konstitution Der Mischkristalle Und Die Raumfüllung Der Atome. Zeitschrift für Phys. 1921, 5 (1), 17-26.

(26) Denton, A. R.; Ashcroft, N. W. Vegard’s Law. Phys. Rev. A 1991, 43 (6), 3161-3164.

(27) Crawley, A. F. Densities of Liquid Metals and Alloys. Int. Metall. Rev. 1974, 19 (1), 32-48.

(28) Cowley, J. M. An Approximate Theory of Order in Alloys. Phys. Rev. 1950, 77 (5), 669-675.

(29) Norman, N.; Warren, B. E. X-Ray Measurement of Short Range Order in Ag-Au. J. Appl. Phys. 1951, 22 (4), 483-486.

(30) Kostiuchenko, T.; Körmann, F.; Neugebauer, J.; Shapeev, A. Impact of Lattice Relaxations on Phase Transitions in a High-Entropy Alloy Studied by Machine-Learning Potentials. npj Comput. Mater. 2019, 5 (1), 55.

(31) Kostiuchenko, T.; Ruban, A. V; Neugebauer, J.; Shapeev, A.; Körmann, F. Short-Range Order in Face-Centered Cubic VCoNi Alloys. Phys. Rev. Mater. 2020, 4 (11), 113802.

(32) Schönfeld, B.; Sax, C. R.; Zemp, J.; Engelke, M.; Boesecke, P.; Kresse, T.; Boll, T.; Al-Kassab, T.; Peil, O. E.; Ruban, A. V. Local Order in Cr-Fe-Co-Ni: Experiment and Electronic Structure Calculations. Phys. Rev. B 2019, 99 (1), 14206. 
(33) Wang, J.; Wang, S.-Q. Surface Energy and Work Function of Fcc and Bcc Crystals: Density Functional Study. Surf. Sci. 2014, 630, 216-224.

(34) Knudsen, J.; Nilekar, A. U.; Vang, R. T.; Schnadt, J.; Kunkes, E. L.; Dumesic, J. A.; Mavrikakis, M.; Besenbacher, F. A Cu/Pt Near-Surface Alloy for Water-Gas Shift Catalysis. J. Am. Chem. Soc. 2007, 129 (20), 6485-6490.

(35) Stephens, I. E. L.; Bondarenko, A. S.; Perez-Alonso, F. J.; Calle-Vallejo, F.; Bech, L.; Johansson, T. P.; Jepsen, A. K.; Frydendal, R.; Knudsen, B. P.; Rossmeisl, J.; et al. Tuning the Activity of Pt(111) for Oxygen Electroreduction by Subsurface Alloying. J. Am. Chem. Soc. 2011, 133 (14), 5485-5491.

(36) Wynblatt, P.; Chatain, D. Modeling Grain Boundary and Surface Segregation in Multicomponent High-Entropy Alloys. Phys. Rev. Mater. 2019, 3 (5), 54004.

(37) Ferrari, A.; Körmann, F. Surface Segregation in Cr-Mn-Fe-Co-Ni High Entropy Alloys. Appl. Surf. Sci. 2020, 533, 147471.

(38) Andersen, M.; Medford, A. J.; Nørskov, J. K.; Reuter, K. Scaling-Relation-Based Analysis of Bifunctional Catalysis: The Case for Homogeneous Bimetallic Alloys. ACS Catal. 2017, 7 (6), 3960-3967.

(39) Yan, D.; Kristoffersen, H. H.; Pedersen, J. K.; Rossmeisl, J. Rationally Tailoring Catalysts for the CO Oxidation Reaction by Using DFT Calculations. ACS Catal. 2022, 12, 116-125. 